\title{
Tagebuchstudie zu Activity-Based Flexible Offices
}

\section{Wie das Zusammenspiel von Arbeitsaufgabe und Arbeitsort das psychische Wohlbefinden fördert}

\author{
Cathrin Becker ${ }^{1}$, Roman Soucek ${ }^{2}$ (D), Jennifer Gunkel ${ }^{3,4}$, Sarah Lütke Lanfer ${ }^{1}$ \\ und Anja S. Göritz ${ }^{1}$ \\ ${ }^{1}$ Albert-Ludwigs-Universität Freiburg \\ ${ }^{2}$ Friedrich-Alexander-Universität Erlangen-Nürnberg \\ ${ }^{3}$ rheform Workplacelnnovation GmbH, München \\ ${ }^{4}$ Hochschule Fresenius für Management, Wirtschaft \& Medien GmbH, München
}

\begin{abstract}
Zusammenfassung: Die Digitalisierung und Globalisierung fordern von Unternehmen vermehrte Flexibilität, was sich in der Gestaltung von Bürokonzepten niederschlägt. Es entstehen häufig Activity-Based Flexible Offices, die sich durch ein offenes und flexibles Raumkonzept auszeichnen. Dabei befindet sich ein Großteil der Arbeitsplätze in offenen Bereichen ohne Zwischenwände und ohne fest zugewiesene Arbeitsplätze. Dieses Konzept ist für den Austausch ausgelegt, bietet aber auch Rückzugsmöglichkeiten wie etwa Konzentrationszellen. In drei international agierenden Unternehmen wurde eine webbasierte Tagebuchstudie durchgeführt, die den Einfluss der aufgabenbezogenen Konzentrationserfordernisse und des Arbeitsortes auf die wahrgenommene Passung zwischen Arbeitsaufgabe und Arbeitsort sowie das psychische Wohlbefinden untersuchte. Die Ergebnisse zeigen, dass offene Arbeitsbereiche bei Aufgaben mit hohen Konzentrationserfordernissen als nicht passend wahrgenommen werden, jedoch nicht im Homeoffice. Generell geht die Passung zwischen Arbeitsaufgabe und Arbeitsort mit dem psychischen Wohlbefinden der Beschäftigten einher. Zusammenfassend sollte das Activity-Based Flexible Office als ganzheitliches Konzept mit Rückzugsmöglichkeiten innerhalb als auch außerhalb des Unternehmens verstanden werden.
\end{abstract}

Schlüsselwörter: Activity-Based Flexible Offices, Homeoffice, Konzentration, Task-Environment-Fit, psychisches Wohlbefinden

Diary Study on Activity-Based Flexible Offices: How the Interplay Between Work Task and Place of Work Promotes Psychological Well-Being

Abstract: Digitization and globalization require greater flexibility from companies, which in turn affects the conceptualization and design of their offices. Activity-based flexible offices have been recently evolving, which are characterized by an open and flexible arrangement of different workspaces, most of which are located in open spaces without intermediate walls and without assigned workplaces. These workspaces are designed to enable exchange, but also to provide places for withdrawal, such as concentration cells. We conducted a webbased diary study in three international companies and examined the joint influence of task-related concentration requirements and the workplace on perceived task-environment fit and psychological well-being. The results revealed that open workspaces are perceived as less suitable for tasks that demand great concentration, though this does not apply to the home office. Furthermore, task-environment fit was associated with psychological well-being. In summary, the concept of activity-based flexible offices should be interpreted holistically and serve to provide places of retreat both inside and outside the company.

Keywords: activity-based flexible office, home office, concentration requirements, task-environment fit, psychological well-being

Die Digitalisierung und Globalisierung der Arbeitswelt beeinflussen die Büroarbeit in weitreichendem Maße (u. a. Cascio \& Montealegre, 2016; Davis, Leach \& Clegg, 2011). Das Arbeiten in einer digitalisierten und entgrenzten Arbeitswelt wird schneller, komplexer und flexibler (Thörel, Pauls \& Göritz, 2020). Diese Flexibilitätsanforderungen an die Unternehmen spiegeln sich in den Bürokonzepten wider: Wenn Unternehmen derzeit Büroräume umgestalten oder neu entwerfen, dann entstehen häufig offene, flexible Büroflächen (Kratzer, 2017). Hierbei handelt es sich um ein Bürokonzept mit verschiedenen Arbeitsorten für unterschiedliche Arbeitsaufgaben, das oft als „Activity-Based Flexible Office“ bezeichnet und als Synonym für offene Büroflächen bzw. Open Space Büros verwendet wird. Eine zentrale Annahme dieses Konzeptes besteht darin, den Beschäftigten zusätzliche, anforde- 
rungsorientierte Arbeitsflächen bzw. -räume zur Verfügung zu stellen. Activity-Based Flexible Offices gelten als Antwort auf eine beschleunigte Innovationsdynamik, die wachsenden Flexibilitätserfordernisse sowie die Informationsflut einer digitalisierten Wissensgesellschaft (Kratzer, 2017).

Allerdings existieren trotz zunehmender Verbreitung dieses Bürokonzept nur wenige empirische Studien, welche sich mit den psychischen Auswirkungen beschäftigen (Lütke Lanfer \& Pauls, 2017; Lütke Lanfer, Becker \& Göritz, 2021; Wohlers \& Hertel, 2017). In der Forschung wurden bisher vielmehr die Auswirkungen auf die körperliche Gesundheit der Beschäftigten untersucht (Lütke Lanfer \& Pauls, 2017). Im Hinblick auf offene Büros wurde vor negativen Auswirkungen gewarnt (Lärm, Unterbrechungen etc., siehe u. a. Utsch, 2017; Bundesanstalt für Arbeitsschutz und Arbeitsmedizin, 2016), die sich vor allem dann zeigen können, wenn die Tätigkeit hohe Konzentration erfordert. In diesem Zusammenhang stellt sich die Frage, ob die modernen, flexiblen Büros eine negative Auswirkung auf das psychische und physische Wohlbefinden sowie die Leistung der Mitarbeitenden haben (zur negativen Wirkung von Großraumbüros siehe z.B. Herbig, Schneider \& Nowak, 2016).

Der vorliegende Beitrag trägt in verschiedener Hinsicht zum Erkenntnisgewinn bei. Das Augenmerk liegt auf flexiblen Arbeitsplatzkonzepten, in denen die Mitarbeitenden keinen eigenen Arbeitsplatz haben. Nach einer Vorstellung der Besonderheiten von „Activity-Based Flexible Offices" wird untersucht, inwieweit die unterschiedlichen Arbeitsorte in Activity-Based Flexible Offices als passend für konzentriertes Arbeiten wahrgenommen werden. Wir untersuchen diese Frage auf der Ebene einzelner Arbeitsaufgaben und betrachten das Zusammenspiel von Konzentrationserfordernissen und konkreten Arbeitsorten im Rahmen einer Tagebuchstudie sowie den Einfluss auf das psychische Wohlbefinden. Ferner gehen wir der Frage nach, ob der Zusammenhang zwischen Arbeitsaufgaben und dem Wohlbefinden infolge der wahrgenommenen Passung von Arbeitsaufgabe und Arbeitsort erklärt werden kann.

\section{Activity-Based Flexible Offices}

Das Activity-Based Flexible Office stellt ein modernes Büro- und Arbeitskonzept dar, das folgende Kriterien erfüllt: a) Die Arbeitsumgebung besteht aus einer Vielfalt an unterschiedlichen Räumlichkeiten, die unterschiedliche Arbeitsaktivitäten unterstützen und somit aktivitätsbasiertes Arbeiten ermöglichen, b) die individuellen Arbeitsplätze befinden sich in der Regel in offen gehaltenen „Open Space“-Zonen und c) die individuellen Arbeits- plätze sind nicht einer Person zugeordnet, sondern jeder Arbeitsplatz kann potenziell von jedem Mitarbeitenden genutzt werden (Gerdenitsch, Korunka \& Hertl, 2018; Bodin Danielsson \& Bodin, 2008).

Activity-Based Flexible Offices bestehen nicht nur aus vielen einzelnen Arbeitsplätzen, sondern verfügen über zusätzliche Bereiche bzw. Arbeitsoptionen, wie etwa Meetingpoints, an denen sich die Mitarbeitenden austauschen können. Weitere Beispiele sind Think Tanks bzw. Konzentrationszellen, d.h. schallisolierte Räume für konzentriertes Arbeiten, oder Telefonzellen, in denen Mitarbeitende abgeschirmt telefonieren können. Die funktionale Abgrenzung in unterschiedliche Bereiche erfolgt über Zonierungen und Mobiliar, nicht durch Wände bzw. Mauern (Stadler, 2011). Activity-Based Flexible Offices sollen den Beschäftigten eine Vielfalt an unterschiedlichen Arbeitsbereichen und -zonen zur Verfügung stellen, um verschiedenen Aufgabenanforderungen gerecht zu werden (z.B. Wohlers \& Hertel, 2017). Je nach Arbeitsaufgabe kann eine bestimmte Umgebung genutzt werden. Beispielsweise kann für ein Telefonat anstatt des offenen Arbeitsbereichs ein Besprechungsraum oder eine Telefonzelle genutzt werden; dadurch werden die Kollegen nicht gestört und die telefonierende Person hat die Gelegenheit, vertrauliche Gespräche zu führen. Diese Rückzugsmöglichkeiten sollen konzentriertes Arbeiten ermöglichen und die Nachteile von geteilten und offenen Bürokonzepten auffangen, die beispielsweise durch häufigere Ablenkungen im Vergleich zu Einzelbüros gekennzeichnet sind (Seddigh, Berntson, Bodin Danielson \& Westerlund, 2014).

Zusammengefasst können traditionelle Konzepte mit festen Arbeitsplätzen den wechselnden Anforderungen der Arbeitsaufgaben nicht gerecht werden. Deswegen besteht eine zentrale Annahme bei Activity-Based Flexible Offices darin, verschiedene Arbeitsplätze anzubieten und die Beschäftigten zu befähigen, ihren Arbeitsplatz entsprechend ihrer aktuellen Aufgabe auszuwählen (AppelMeulenbroek, Groenen \& Janssen, 2011). Dadurch erhöhen sie die Passung zwischen Arbeitsaufgabe und Arbeitsort. Diese Passung kann als Form des Person-Environment-Fit verstanden werden (z. B. Caplan, 1987).

\section{Passung zwischen Arbeitsaufgabe und Arbeitsort}

Die Person-Environment-Fit-Theorie (Caplan, 1987; Edwards, Cable, Williamson, Lambert \& Shipp, 2006; Kristof, 1996) nimmt an, dass eine Übereinstimmung zwischen den Bedürfnissen einer Person und den Merkmalen der Umwelt in psychischem Wohlbefinden, Arbeitszufriedenheit, hoher Motivation und hoher Leistung resultieren 
(Edwards, Caplan \& Harrison, 1998; Wohlers \& Hertel, 2017). Analog nehmen wir an, dass eine hohe Passung zwischen Aufgabenerfordernissen und Arbeitsort zu ähnlichen Resultaten führt. Dementsprechend interpretieren wir die Passung zwischen aktueller Arbeitsaufgabe und aktuellem Arbeitsort als Task-Environment-Fit.

Moderne Bürokonzepte ermöglichen es, die Örtlichkeit den Erfordernissen der aktuellen Arbeitsaufgabe anzupassen und schaffen dadurch eine unterstützende Umgebung im Sinne eines Task-Environment-Fits (Wohlers \& Hertel, 2017; Wohlers, Hartner-Tiefenthaler \& Hertel, 2017). Die Frage nach der Passung zwischen Arbeitsaufgabe und Arbeitsort wurde bislang vorwiegend auf der Ebene der allgemeinen Arbeitstätigkeit untersucht, also etwa hinsichtlich einer adäquaten Ausstattung des Arbeitsplatzes durch den Arbeitgeber. Bei Activity-Based Flexible Offices stellt der Arbeitgeber viele unterschiedliche Arbeitsorte zur Verfügung; die Wahl eines passenden Arbeitsortes trifft der Beschäftigte jedoch selbst. Diese Entscheidung wird auf der Grundlage einzelner Arbeitsaufgaben getroffen, die sich im Laufe des Tages ändern können und mit wechselnden Erfordernissen einhergehen können. Diese fortlaufende Anpassung des Arbeitsortes an die jeweiligen Anforderungen wurde empirisch kaum untersucht (vgl. Seddigh et al., 2014) bzw. lediglich in querschnittlichen Untersuchungen aufgezeigt (Wohlers et al., 2017).

Hinsichtlich der Zufriedenheit mit verschiedenen Aspekten von Activity-Based Flexible Offices wie etwa der Akustik oder Rückzugsmöglichkeiten zeigen sich uneinheitliche Ergebnisse (Becker, Lütke Lanfer \& Kratzer, 2019; Kratzer, 2020). Diese Varianz in der Zufriedenheit spiegelt zum einen die unterschiedliche Ausgestaltung von Activity-Based Flexible Offices bei unterschiedlichen Unternehmen wider, könnte aber auch auf sich verändernde Erfordernisse der jeweiligen Arbeitsaktivitäten zurückzuführen sein. In einer Literaturübersicht kommen Engelen et al. (2018) zum Schluss, dass Activity-Based Working in den meisten Studien als negativ eingeschätzt wird. In flexiblen Büroräumen wurde u.a. die Konzentration als schlechter eingeschätzt als bei anderen Bürotypen (de Been \& Beijer, 2014). Ebenso berichteten Beschäftigte in geteilten und offenen Bürokonzepten von mehr Ablenkungen im Vergleich zu solchen in Einzelbüros (Seddigh et al., 2014; Elsbach \& Pratt, 2007). Dem entsprechend nehmen wir an, dass bei Arbeitsaufgaben mit hohen Konzentrationserfordernissen offene Arbeitsbereiche als weniger passend eingeschätzt werden.

Hypothese 1: Bei Arbeitsaufgaben mit höheren Konzentrationserfordernissen ist an einem Arbeitsplatz im offenen Bereich die empfundene Passung zwischen Arbeitsaufgabe und Arbeitsort niedriger ausgeprägt.
In Activity-Based Flexible Offices befinden sich sowohl Bereiche für konzentriertes als auch für interaktives Arbeiten (Bodin Danielsson \& Bodin 2008; Bodin Danielsson, Singh Chungkham, Wulff \& Westerlund, 2014; Heerwagen, Kampschroer, Powell \& Loftness, 2004). Diese Bürogestaltung bietet den Beschäftigten die Möglichkeit, mehr Kontrolle über akustische Störungen und Unterbrechungen zu erlangen (Wohlers \& Hertel, 2017) sowie individuelle und aufgabenbezogene Bedürfnisse beim Arbeiten zu berücksichtigen (Kratzer, 2017). Vor diesem Hintergrund sollte die Passung bei Arbeitsaufgaben, die eine hohe Konzentration erfordern, an Rückzugsorten als besser eingeschätzt werden. Dies sollte für innerbetriebliche Rückzugsorte - etwa Konzentrationszellen - zutreffen als auch für Arbeitsorte außerhalb des Betriebs wie im Homeoffice. Insbesondere gibt es im Homeoffice weniger Unterbrechungen durch Kollegen (Van der Voordt, 2004).

Hypothese 2: Bei Arbeitsaufgaben mit höheren Konzentrationserfordernissen ist an einem Rückzugsort innerhalb des Open-Space-Konzeptes im Firmengebäude die empfundene Passung zwischen Arbeitsaufgabe und -ort höher ausgeprägt.

Hypothese 3: Bei Arbeitsaufgaben mit höheren Konzentrationserfordernissen ist im Homeoffice die empfundene Passung zwischen Arbeitsaufgabe und -ort höher ausgeprägt.

\section{Arbeitsaufgabe, Arbeitsort und psychisches Wohlbefinden}

Die Arbeitsumgebung beeinflusst das psychische Wohlbefinden, die Leistungsfähigkeit sowie die Gesundheit von Beschäftigten (Windlinger, Konkol, Schanné, Sesboüé \& Neck, 2014; Richter \& Cernavin 2016; Rothe et al. 2017). Bei der Frage nach den Auswirkungen von Activity-Based Flexible Offices auf die psychische Gesundheit liegt das psychische Wohlbefinden der Beschäftigten im Augenmerk: Ein passender Arbeitsraum erhöht das Wohlbefinden und Wohlbefinden ist wiederum gut für die Gesundheit (Kratzer, 2017).

Zur Frage der Wirkung von Bürostrukturen auf die Gesundheit identifizierten De Croon, Sluiter, Kuijer und Frings-Dresen (2005) in einem systematischen Review 49 Studien: Es zeigten sich inkonsistente Befunde mit Blick auf kurz- und langfristige Folgen. In einer systematischen Literaturübersicht kamen Lütke Lanfer und Pauls (2017) zu einem ähnlichen Ergebnis: Mit Blick auf kurzfristige und langfristige psychische Folgen (z. B. Erschöpfung bzw. psychisches Wohlbefinden) konnten die früheren Ergebnisse von De Croon et al. (2005) untermauert werden.

Eine Erklärung für diese inkonsistenten Ergebnisse könnte sein, dass in den Studien keine spezifischen Ar- 


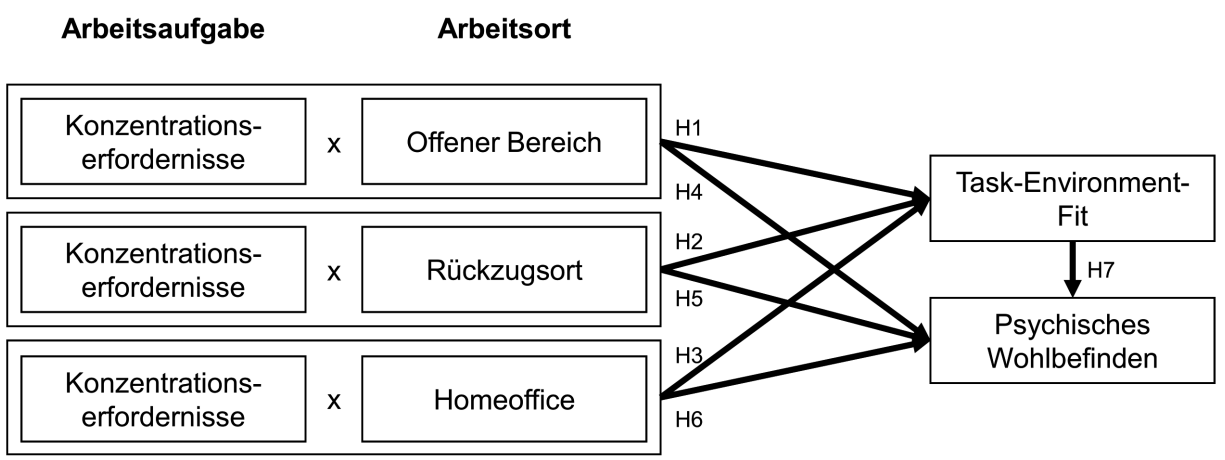

Abbildung 1. Übersicht der Hypothesen.

beitsorte (in Form von Raummodulen) betrachtet wurden. Dementsprechend nehmen wir an, dass Aufgaben, die ein konzentriertes Arbeiten erfordern, in offenen Bereichen des Activity-Based Flexible Office mit einem geringen psychischen Wohlbefinden einhergehen, wobei das Wohlbefinden an diversen Rückzugsorten wie Konzentrationszelle aber auch Homeoffice, höher ausgeprägt ist.

Hypothese 4: Bei Arbeitsaufgaben mit höheren Konzentrationserfordernissen ist an einem Arbeitsplatz im offenen Bereich das psychische Wohlbefinden niedriger ausgeprägt.

Hypothese 5: Bei Arbeitsaufgaben mit höheren Konzentrationserfordernissen ist an einem Rückzugsort innerhalb des Open-Space-Konzeptes im Firmengebäude das psychische Wohlbefinden höher ausgeprägt.

Hypothese 6: Bei Arbeitsaufgaben mit höheren Konzentrationserfordernissen ist im Homeoffice das psychische Wohlbefinden höher ausgeprägt.

\section{Passung und psychisches Wohlbefinden}

Eine hohe Passung zwischen Aufgabenerfordernissen und Arbeitsort kann als spezifische Form des Needs-SuppliesFit - d.h. der Passung zwischen Bedürfnissen des Mitarbeitenden und den Ressourcen der Arbeitsumwelt - verstanden werden. Gerdenitsch et al. (2018) zeigten in einer Längsschnittstudie, dass eine solche Passung von individuellen Bedürfnissen und Ressourcen der Arbeitsumwelt den positiven Zusammenhang zwischen einem Open Space Konzept und Arbeitszufriedenheit stärken kann. Wohlers und Hertel (2017) postulieren zudem, dass ein hoher Task-Environment-Fit negative Effekte akustischer Unterbrechungen und Ablenkungen auf das psychische Wohlbefinden der Beschäftigten mindere. Folglich nehmen wir an, dass die wahrgenommene Passung mit dem Wohlbefinden einhergeht.

Hypothese 7: Je höher die empfundene Passung zwischen Arbeitsaufgabe und -ort ist, desto höher ist das psychische Wohlbefinden.
Abbildung 1 fasst die Hypothesen zusammen. Insgesamt nehmen wir an, dass bei Arbeitsaufgaben, die ein hohes Konzentrationserfordernis aufweisen und im offenen Bereich bearbeitet werden, mit einem geringen TaskEnvironment-Fit (Hypothese 1) sowie Wohlbefinden einhergehen (Hypothese 4). Dem gegenüber ist bei hohen Konzentrationserfordernissen in Rückzugsorten (z. B. Konzentrationszellen) und im Homeoffice der Task-Environment-Fit (Hypothesen 2 und 3) sowie das Wohlbefinden (Hypothesen 5 und 6) gegeben. Eine hohe Passung zwischen Arbeitsaufgabe und Arbeitsort geht schließlich mit dem Wohlbefinden einher (Hypothese 7).

\section{Methode}

\section{Stichprobe}

Die Befragung erfolgte in drei international agierenden Unternehmen, darunter zwei Unternehmen aus der Beratungs- bzw. Dienstleistungsbranche und einem Unternehmen aus der Luftfahrtbranche. Die befragten Beschäftigten dieser Unternehmen arbeiteten an Standorten in Deutschland. Die Stichprobe bestand aus 62 Beschäftigten, die sich an der Vorerhebung und mindestens einer weiteren Erhebung beteiligten. Von den 62 Beschäftigten waren $32 \%$ männlich. Der Altersdurchschnitt lag bei 36.95 Jahren $(S D=10.00)$. Die durchschnittliche Arbeitszeit betrug 37.98 Stunden pro Woche $(S D=5.50)$. Die Untersuchungsteilnehmer wurden unternehmensintern im Schneeballsystem rekrutiert. Insgesamt nahmen 36 Personen an 4 bzw. 5 Tagen an der Befragung teil.

Es wurden nur Personen berücksichtigt, die im Rahmen eines flexiblen Arbeitsplatzkonzeptes ohne einen fest zugewiesenen Arbeitsplatz beschäftigt waren und in der Befragung als Arbeitsort „Open-Space-Büro“, „Konzentrationszelle“ oder „Homeoffice“ angegeben hatten. Weitere Arbeitsorte wie etwa beim Kunden vor Ort wurden in dieser Studie nicht berücksichtigt. Die Arbeitsplatzkon- 
zepte der betrachteten Unternehmen sind in der Art der Zusammenstellung der Räume und in Bezug auf die Grundkonzepte der Büroarbeit (Arbeiten ist von überall möglich, vielfältige Arbeitsorte, keine festen Plätze) ähnlich und erfüllen alle die Kriterien eines Activity-Based Flexible Offices.

\section{Ablauf der Untersuchung}

Die Untersuchung wurde als webbasierte Tagebuchbefragung durchgeführt und erstreckte sich über zwei Wochen. In der ersten Woche erhielten die Personen via EMail den Zugangslink zum ersten Fragebogen, der neben soziodemografischen Daten, Merkmale des Unternehmens (z.B. Beschäftigtenzahl und Arbeitsplatzkonzept) erfasste. Zu Beginn der zweiten Woche wurde der Link zum zweiten Fragebogen versandt, welcher tagesbezogene Arbeitsunterbrechungen und Konzentrationsanforderungen sowie aufgabenbezogene Konzentrationserfordernisse, Arbeitsort, die wahrgenommene Passung zwischen Arbeitsaufgabe und Arbeitsort sowie das psychische Wohlbefinden erfasste. Dieser Fragebogen wurde in der zweiten Woche gegen Ende jedes der fünf Arbeitstage von den Teilnehmenden ausgefüllt.

\section{Operationalisierung der Variablen}

Arbeitsort. Die Teilnehmer wurden gefragt, an welchem Arbeitsort sie die letzten zwei Stunden gearbeitet hatten („Wo haben Sie in den vergangenen zwei Stunden vorrangig gearbeitet?"). Als Antwortoptionen standen zur Verfügung: offener Arbeitsplatz im Activity-Based Flexible Office, Besprechungsraum/Konferenzraum, Konzentrationszelle, Telefonzelle, Kreativraum bzw. Projektraum, Teeküche, Sitzecke, vor Ort beim Kunden und Homeoffice. Für die Auswertung wurden nur die Arbeitsorte Arbeitsplatz im offenen Bereich, Konzentrationszelle und Homeoffice berücksichtigt; die weiteren Arbeitsorte wiesen eine geringere Nutzungshäufigkeit auf und wurden deshalb nicht berücksichtig. Der Arbeitsort wurde dummy-kodiert, wobei Arbeitsplatz im offenen Bereich als Gruppe mit den häufigsten Nennungen als Referenzkategorie verwendet wurde. Diese Kodierung resultiert in zwei dummy-kodierten Variablen Konzentrationszelle und Homeoffice, die jeweils den Unterschied zum offenen Bereich abbilden.

Aufgabenbezogene Konzentration. Die Teilnehmenden wurden gefragt, welche Aufgabe sie in den letzten zwei Stunden vorrangig bearbeitet hatten und in welchem Ausmaß diese Aufgabe ihre Konzentration erforderte („In welchem Ausmaß beinhaltete diese Aufgabe folgende
Anforderungen? Konzentration"). Dieses aufgabenbezogene Konzentrationserfordernis wurde auf einer LikertSkala beantwortet, die von 1 (in sehr niedrigem Ausmaß) bis 7 (in sehr hohem Ausmaß) reichte.

Task-Environment-Fit. Der Task-Environment-Fit wurde mit dem Item „Wie geeignet empfanden Sie den genannten Arbeitsort für diese Aufgabe?" erhoben. Das Item wurde anhand einer Skala von 1 (gar nicht geeignet) bis 7 (völlig geeignet) beantwortet.

Psychisches Wohlbefinden. Dieses Konstrukt wurde erfasst anhand der deutschen Version des WHO-5-Wohlbefindensindex (Brähler, Mühlan, Albani \& Schmidt, 2007). Der Index besteht aus fünf Items, die auf einer Skala von 1 (zu keinem Zeitpunkt) bis 6 (die ganze Zeit) beantwortet wurden. In der Originalfassung bezieht sich der WHO-5 auf einen Zeitraum von zwei Wochen. Für die Befragung wurde er angepasst und fragte nach dem gegenwärtigen Tag (z.B. „Heute war ich froh und guter Laune.“). Das Cronbachs $\alpha$ betrug .75 .

Kontrollvariablen. Als Kontrollvariablen wurden Geschlecht und Alter erhoben sowie die wöchentliche Arbeitszeit in Stunden. Darüber hinaus wurden tagesbezogene Konzentrationsanforderungen und das Ausmaß an Arbeitsunterbrechungen erfragt. Die tagesbezogenen Konzentrationsanforderungen wurden mithilfe einer Skala des ISTA (Semmer, Zapf \& Dunckel, 1999) erhoben und bestanden aus fünf Items, deren zeitlicher Kontext auf den gegenwärtigen Tag angepasst wurde (z. B. „Wie oft mussten Sie heute bei Ihrer Arbeit viele Dinge gleichzeitig im Kopf haben?"). Die Einschätzung erfolgte auf einer Rating-Skala von 1 (nie) bis 5 (mehrmals pro Stunde). Cronbachs a betrug .73. Das tagesbezogene Ausmaß der Arbeitsunterbrechungen wurde mit drei Items auf einer Rating-Skala von 1 (nie) bis 5 (mehrmals pro Stunde) erfasst (Semmer et al., 1999). Cronbachs a lag bei .77.

\section{Analyseverfahren}

Die Auswertung erfolgte mit linearen gemischten Modellen für Längsschnittdaten (multilevel model for change, Singer \& Willett, 2003). Die Zeit wurde in Tagen abgebildet und als linearer sowie quadratischer Effekt bei der Auswertung berücksichtigt. Der indirekte Effekt wurde nach dem Vorgehen von Imai, Keele und Tingley (2010) überprüft.

\section{Ergebnisse}

Hinsichtlich des Arbeitsortes gaben die Befragten an, $74 \%$ ihrer Arbeitszeit an einem offenen Arbeitsplatz im 
Activity-Based Flexible Office zu verbringen. Als andere Arbeitsorte wurden Konzentrationszellen (9\%) sowie das Homeoffice (17\%) genannt. In Tabelle 1 sind die Mittelwerte, Standardabweichungen und Interkorrelationen der erhobenen Variablen über alle Messzeitpunkte aufgeführt.

\section{Passung zwischen Arbeitsaufgabe und Arbeitsort}

In Tabelle 2 wird der Einfluss des Arbeitsortes und des aufgabenbezogenen Konzentrationserfordernisses auf die wahrgenommene Passung zwischen der Arbeitsaufgabe und dem Arbeitsort dargestellt. In Modell 1a wurden neben demografischen Angaben die wöchentliche Arbeitszeit und die Konzentrationsanforderungen und Arbeitsunterbrechungen des aktuellen Arbeitstages als Kontrollvariablen berücksichtigt. Hinsichtlich des Arbeitsortes fällt auf, dass Homeoffice im Vergleich zu offenen Arbeitsplätzen im Activity-Based Flexible Office als passender wahrgenommen wird: $B=1.45, p<.001$. In Modell $1 \mathrm{~b}$ wurden die aufgabenbezogenen Konzentrationserfordernisse aufgenommen. Die Ergebnisse von Modell $1 \mathrm{~b}$ zeigen, dass hohe aufgabenbezogene Erfordernisse an die Konzentration mit einem geringeren Task-EnvironmentFit - unabhängig vom Arbeitsort - einhergehen: $B=-0.34$, $p<.001$.

In Modell 1c wurden zusätzlich die Interaktionen zwischen den Konzentrationserfordernissen der Arbeitsaufgabe und den Arbeitsorten aufgenommen. Da der offene Arbeitsplatz im Activity-Based Flexible Office als Referenzarbeitsort herangezogen wurde und die Interaktionseffekte die beiden anderen Arbeitsorte der Konzentrationszelle und des Homeoffice enthalten, gibt der Haupteffekt der aufgabenbezogenen Konzentrationserfordernisse den Einfluss für den Fall des offenen Arbeitsplatzes in Activity-Based Flexible Office an, welcher negativ ausfällt, $B=-0.51, p<.001$. Demnach wird die Passung von offenen Arbeitsplätzen in Activity-Based Flexible Offices bei hohen aufgabenbezogenen Konzentrationserfordernissen als geringer empfunden, wodurch Hypothese 1 bestätigt wird. Der Interaktionseffekt aus Konzentrationserfordernis und dem Arbeitsort Konzentrationszelle ist nicht signifikant, $B=-0.19, p=.559$, so dass die Passung der Konzentrationszelle im Vergleich zum offenen Arbeitsplatz im Activity-Based Flexible Office im Falle hoher aufgabenbezogener Konzentrationserfordernisse nicht anders eingeschätzt wird. Hypothese 2 konnte damit nicht bestätigt werden. Im Fall von Homeoffice ergibt sich ein positiver Interaktionseffekt mit Konzentrationserfordernissen der Aufgabe: $B=0.58, p<.001$. Das Homeoffice wird im Vergleich zum offenen Arbeitsplatz im Acti-

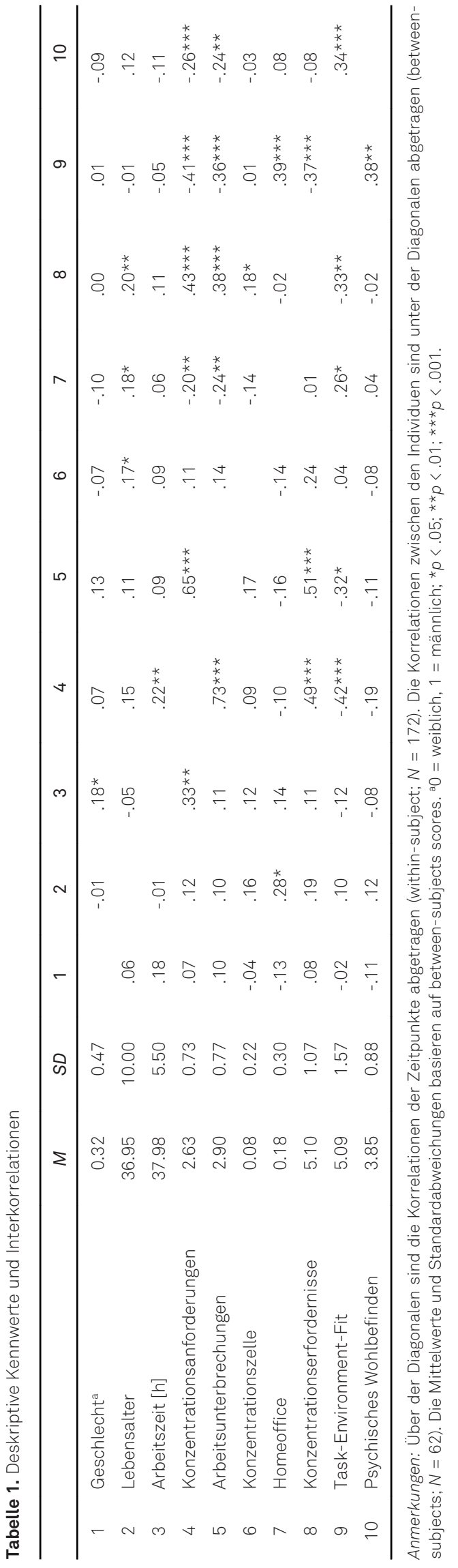


Tabelle 2. Einfluss des Arbeitsortes und der aufgabenbezogenen Konzentrationserfordernisse auf den Task-Environment-Fit

\begin{tabular}{|c|c|c|c|}
\hline & Modell 1a & Modell 1b & Modell 1c \\
\hline & & Fixed effects & \\
\hline (Intercept) & $7.85 * * *$ & $7.22 * * *$ & $7.08 * * *$ \\
\hline \multicolumn{4}{|l|}{ Level 1 (within subjects) } \\
\hline Konzentrationsanforderungen & $-0.47 \star \star$ & -0.31 & -0.31 \\
\hline Arbeitsunterbrechungen & $-0.32 \star$ & $-0.30 *$ & -0.26 \\
\hline Zeit & -0.29 & -0.28 & -0.34 \\
\hline Zeit $\times$ Zeit & 0.06 & 0.05 & 0.06 \\
\hline Konzentrationszelle & 0.30 & 0.44 & 0.69 \\
\hline Homeoffice & $1.45 * \star \star$ & $1.53 * * \star$ & $1.55^{* * *}$ \\
\hline Konzentrationserfordernisse & & $-0.34 * * \star$ & $-0.51 * * *$ \\
\hline Konz.erfordernisse $\times$ Konz.zelle & & & -0.19 \\
\hline Konz.erfordernisse $x$ Homeoffice & & & $0.58 * * *$ \\
\hline \multicolumn{4}{|l|}{ Level 2 (between subjects) } \\
\hline Geschlecht $^{\mathrm{a}}$ & 0.24 & 0.26 & 0.21 \\
\hline Lebensalter & 0.01 & 0.01 & 0.01 \\
\hline \multirow[t]{2}{*}{ Arbeitszeit } & -0.02 & -0.03 & -0.03 \\
\hline & & Random effects & \\
\hline \multicolumn{4}{|l|}{ Level 1 (within subjects) } \\
\hline Residual variance $\left(\sigma^{2}\right)$ & $0.90 * * *$ & $0.85^{\star \star \star}$ & $0.69 * * *$ \\
\hline \multicolumn{4}{|l|}{ Level 2 (between subjects) } \\
\hline Intercept/Intercept $\tau_{00}$ & $1.87 * \star *$ & $1.46^{* \star \star}$ & $1.58 * * *$ \\
\hline Slope/Slope $\tau_{11}$ & $0.05^{\star \star \star}$ & $0.02 * * \star$ & $0.04 * * *$ \\
\hline \multirow[t]{2}{*}{ Intercept/Slope $\rho_{01}$} & -0.13 & -0.03 & -0.07 \\
\hline & & Model fit & \\
\hline Deviance (-2LL) & 586.94 & 571.51 & 556.34 \\
\hline Change in Deviance & & $15.43 * \star \star$ & $15.18 * \star \star$ \\
\hline AlC & 614.94 & 601.51 & 590.34 \\
\hline$B / C$ & 659.00 & 648.73 & 643.84 \\
\hline
\end{tabular}

Anmerkungen: $N=172 ;{ }^{a} 0=$ weiblich, $1=$ männlich; ${ }^{\star} p<.05 ;{ }^{*} p<.01 ;{ }^{* \star} p<.001$.

vity-Based Flexible Office bei hohem Konzentrationserfordernis als passender wahrgenommen, was Hypothese 3 bestätigt.

Dieser Einfluss von Konzentrationserfordernissen und Homeoffice vs. offene Arbeitsplätze im Activity-Based Flexible Office auf den Task-Environment-Fit ist in Abbildung 2 dargestellt. Es ist ersichtlich, dass der negative Einfluss von steigenden Konzentrationserfordernissen auf die Passung im Falle des Arbeitsplatzes im offenen Arbeitsbereich eines Activity-Based Flexible Office deutlich ausgeprägt ist, was auf das Homeoffice nicht zutrifft.

\section{Passung und psychisches Wohlbefinden}

In Tabelle 3 sind die Einflussgrößen auf das psychische Wohlbefinden dargestellt. In Modell 2a wurden neben
Demografika die wöchentliche Arbeitszeit sowie die Konzentrationsanforderungen und Arbeitsunterbrechungen des aktuellen Arbeitstages als Kontrollvariablen berücksichtigt. In Modell $2 \mathrm{~b}$ wird das Konzentrationserfordernis der aktuellen Aufgabe aufgenommen; dieses hat keinen Einfluss auf das psychische Wohlbefinden: $B=-0.02, p=$ .756. Modell $2 \mathrm{c}$ berücksichtigt die Wechselwirkung von Konzentrationserfordernissen und den einzelnen Arbeitsorten. Der Haupteffekt der Konzentrationserfordernisse spiegelt den Einfluss der Konzentrationserfordernisse im Falle des offenen Arbeitsplatzes im Activity-Based Flexible Office wider, welcher nicht signifikant ausfällt, $B=$ $-0.06, p=.403$. Hypothese 4 konnte damit nicht bestätigt werden. Weiterhin übten weder die Konzentrationszelle, $B=0.06, p=.792$, noch das Homeoffice, $B=0.12, p=$ .268 , einen Einfluss auf das Wohlbefinden im Falle höherer aufgabenbezogener Konzentrationserfordernisse aus. Hypothesen 5 und 6 konnten somit nicht bestätigt wer- 


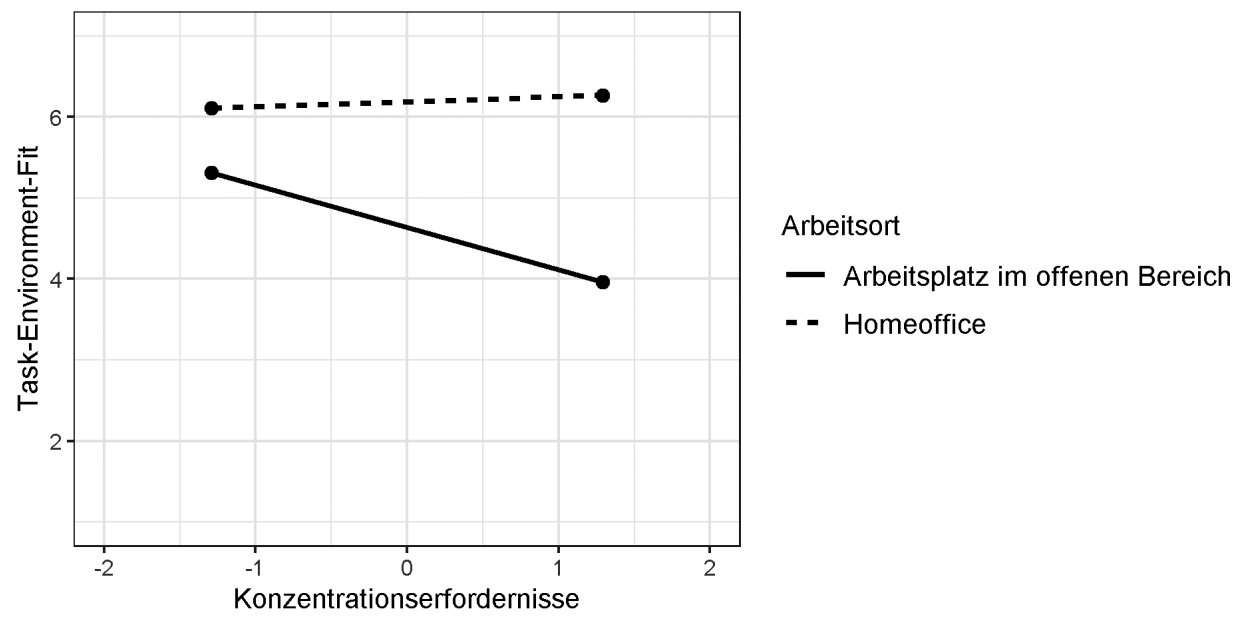

Abbildung 2. Interaktion zwischen Konzentrationserfordernissen und Arbeitsort auf Task-Environment-Fit.

Tabelle 3. Einfluss des Arbeitsortes und der aufgabenbezogenen Konzentrationserfordernisse auf das psychische Wohlbefinden

\begin{tabular}{|c|c|c|c|c|}
\hline & Modell 2a & Modell 2b & Modell 2c & Modell 2d \\
\hline & \multicolumn{4}{|c|}{ Fixed effects } \\
\hline (Intercept) & $4.43 * * *$ & $4.41 * \star \star$ & $4.43 * \star \star$ & $3.12^{\star \star}$ \\
\hline \multicolumn{5}{|l|}{ Level 1 (within subjects) } \\
\hline Konzentrationsanforderungen & $-0.23 *$ & -0.22 & -0.22 & -0.17 \\
\hline Arbeitsunterbrechungen & -0.16 & -0.16 & -0.15 & -0.10 \\
\hline Zeit & -0.06 & -0.06 & -0.08 & -0.03 \\
\hline Zeit x Zeit & 0.03 & 0.03 & 0.03 & 0.02 \\
\hline Konzentrationszelle & -0.09 & -0.08 & -0.09 & -0.25 \\
\hline Homeoffice & -0.05 & -0.05 & -0.04 & -0.31 \\
\hline Konzentrationserfordernisse & & -0.02 & -0.06 & 0.03 \\
\hline Konz.erfordernisse $\times$ Konz.zelle & & & 0.06 & 0.09 \\
\hline Konz.erfordernisse $x$ Homeoffice & & & 0.12 & 0.02 \\
\hline Task-Environment-Fit & & & & $0.17 * \star \star$ \\
\hline \multicolumn{5}{|l|}{ Level 2 (between subjects) } \\
\hline Geschlecht $^{a}$ & -0.20 & -0.20 & -0.20 & -0.24 \\
\hline Lebensalter & 0.01 & 0.01 & 0.01 & 0.02 \\
\hline \multirow[t]{2}{*}{ Arbeitszeit } & -0.00 & -0.00 & -0.00 & 0.00 \\
\hline & \multicolumn{4}{|c|}{ Random effects } \\
\hline \multicolumn{5}{|l|}{ Level 1 (within subjects) } \\
\hline Residual variance $\left(\sigma^{2}\right)$ & $0.44 * \star \star$ & $0.44 * \star \star$ & $0.43 * * *$ & $0.42 * * *$ \\
\hline \multicolumn{5}{|l|}{ Level 2 (between subjects) } \\
\hline Intercept/Intercept $\tau_{00}$ & $0.75 * \star \star$ & $0.77 \star * \star$ & $0.76 * \star \star$ & $0.63 * \star \star$ \\
\hline Slope/Slope $\tau_{11}$ & $0.01 * * *$ & $0.01 * * *$ & $0.01 * \star \star *$ & $0.02 * * *$ \\
\hline \multirow[t]{2}{*}{ Intercept/Slope $\rho_{01}$} & -0.06 & -0.06 & -0.06 & -0.06 \\
\hline & \multicolumn{4}{|c|}{ Model fit } \\
\hline Deviance $(-2 L L)$ & 439.59 & 439.49 & 438.28 & 428.39 \\
\hline Change in Deviance & & 0.09 & 1.21 & $9.89 * *$ \\
\hline AIC & 467.59 & 469.49 & 472.28 & 464.39 \\
\hline$B / C$ & 511.65 & 516.71 & 525.79 & 521.05 \\
\hline
\end{tabular}

Anmerkungen: $N=172 ;{ }^{a} 0$ = weiblich, 1 = männlich; ${ }^{*} p<.05 ;{ }^{* *} p<.01 ;{ }^{* * *} p<.001$. 
den. In Modell $2 \mathrm{~d}$ wurde zusätzlich der Task-Environment-Fit berücksichtigt; dieser hängt positiv mit Wohlbefinden zusammen: $B=0.17, p=.001$. Hypothese 7 wurde damit bestätigt.

Die Kombination der bestätigten Hypothesen 1 und 7 legt einen indirekten Effekt hoher aufgabenbezogener Konzentrationserfordernisse im Falle des offenen $\mathrm{Ar}$ beitsplatzes im Activity-Based Flexible Office auf das Wohlbefinden nahe, wobei der Effekt durch einen geringeren Task-Environment-Fit vermittelt wird. Die Analyse belegte einen solchen indirekten Effekt: $B=-0.07, p=$ .002, CI95[-0.12, -0.02].

\section{Diskussion}

Das Activity-Based Flexible Office gilt als Antwort auf die wachsenden Flexibilitätserfordernisse einer digitalisierten Wissensgesellschaft. Als Gründe für die Einführung von Activity-Based Flexible Offices in Unternehmen werden u.a. Flächenersparnis, Modernität, Ergonomie oder Effizienz genannt (Kratzer, 2017). Activity-Based Flexible Offices sollen die Beschäftigten bei der Erledigung ihrer Arbeitsaufgaben unterstützen, indem unterschiedliche Arbeitsbereiche zur Verfügung gestellt werden, so dass sich die Beschäftigten den zur jeweiligen Aufgabe passenden Arbeitsbereich aussuchen können. Inwieweit Activity-Based Flexible Offices die Beschäftigten tatsächlich darin unterstützen, ist noch wenig erforscht (Gerdenitsch et al., 2018).

Trotz der zunehmenden Verbreitung von Activity-Based Flexible Offices existieren bislang nur wenige empirische Studien, die sich mit den psychischen Folgen neuer Arbeitswelten beschäftigen; hauptsächlich wurden die Auswirkungen auf die physische Gesundheit von Beschäftigten untersucht. Die vorliegende Studie betrachtete das Zusammenwirken von Arbeitsaufgabe und Arbeitsort auf die wahrgenommene Passung von Arbeitsort und Arbeitsaufgabe sowie auf das psychische Wohlbefinden. Die Ergebnisse zeigen, dass zur Erledigung von Arbeitsaufgaben mit hohen Konzentrationserfordernissen der offene Bereich eines Activity-Based Flexible Offices als weniger passend wahrgenommen wird. Konzentriertes Arbeiten ist den vorliegenden Befunden zufolge an Arbeitsplätzen in offenen Bürobereichen weniger gut möglich. Zudem geht die als gering erlebte Passung von offenen Arbeitsplätzen im Activity-Based Flexible Office für die Erledigung konzentrationsträchtiger Aufgaben mit vermindertem Wohlbefinden einher. Die Ergebnisse zeigen außerdem, dass die im Unternehmen eingerichteten Rückzugsorte wie etwa Konzentrationszellen in geringem Ausmaß genutzt werden und darüber hinaus als nicht passend wahrgenommen werden, wenn Aufgaben mit hohen Konzentrationsanforderungen anstehen.

Warum erledigen dann so viele Beschäftigte konzentrationsträchtige Aufgaben im offenen Bereich des Activity-Based Flexible Offices? Mögliche Gründe sind ein Mangel im Angebot von Rückzugsorten, das Fehlen von organisationalen Nutzungsempfehlungen für das ActivityBased Flexible Office, auf Seiten der Beschäftigten der Mangel an Bewusstsein dafür, bei welchen Arbeitsaufgaben es gut ist, den Ort zu wechseln, oder sonstige Gründe, keinen Rückzugsort aufzusuchen, obwohl man sich bei Aufgaben konzentrieren muss (z. B. Bequemlichkeit oder die Befürchtung, etwas zu verpassen).

Viele Unternehmen schreiben sich „Activity-Based Working " auf die Fahne. Die Realität stellt die Umsetzung von Activity-Based Flexible Offices jedoch vor Herausforderungen: Erstens gehen die anfänglichen Konzepte bei wachsenden Beschäftigtenzahlen oft nicht mehr auf: Wenn die Desk Sharing-Rate wächst und somit mehr Mitarbeitende pro Arbeitsplatz im Büro sind, werden in der Regel auch mehr Rückzugsorte benötigt. Es scheint einen Wendepunkt zu geben, ab welchem das Konzept nicht mehr funktioniert, wenn sich zu viele Personen auf einer Fläche aufhalten. Zweitens stellt sich allzu oft im Verlauf von Projekten heraus, dass doch weniger Fläche als gedacht zur Verfügung steht (Kratzer, 2017). Das kann beispielsweise passieren, wenn man zunächst davon ausging, dass Desk Sharing umgesetzt wird, am Ende aber doch viele Mitarbeitende ihren eigenen Arbeitsplatz beanspruchen. Dann wird mehr Fläche für Arbeitsplätze benötigt und der Anteil der unterstützenden Nebenflächen wie z.B. Rückzugsräume verringert sich.

Ein Activity-Based Flexible Office ist nicht nur ein Raumkonzept, sondern eine neue Art zu arbeiten. In dieser neuen Arbeitswelt funktionieren einige Vorgehensweisen nicht mehr so wie in der alten Welt. Gewohnheiten, die bisher zielführend waren, sind im neuen Konzept möglicherweise kontraproduktiv, etwa am offenen Arbeitsplatz zu telefonieren. Wichtig sind hier sowohl die individuelle als auch die kollektive Kompetenz, die neue Arbeitsumwelt zu nutzen. Mitarbeitende und Führungskräfte müssen die Nutzung ihres Activity-Based Flexible Offices neu erlernen. Sie müssen erstens für sich selbst als Individuum Erfahrungen sammeln, welche Räume sich für welche Aufgaben eignen. Führungskräften kommt eine weitere Rolle zu: Sie müssen den Mitarbeitenden in der adäquaten Raumnutzung ein Vorbild sein und diese zur Nutzung unterschiedlicher Räume ermuntern. Zweitens funktioniert eine gemeinsam genutzte Fläche selten ohne Spielregeln oder festgelegte Standards, die gemeinsam erarbeitet, kommuniziert und nachgehalten werden müssen. Zum Beispiel kann ein Team oder eine Abteilung, die einen bestimmten Arbeitsbereich benutzt, festlegen, dass 
in der freien Fläche Stillarbeit stattfindet und alle Gespräche und Telefonate in geschlossenen Räumen stattfinden - welche selbstredend auch vorhanden sein müssen. Werden diese Regeln von allen eingehalten, so kann die Konzentration auch in offenen Bereichen des ActivityBased Flexible Office funktionieren. Spielregeln existieren aber in vielen Fällen überhaupt nicht oder sie geraten schnell in Vergessenheit, weil niemand daran erinnert oder bei der Einführung von neuen Mitarbeitenden vergessen wird, die Regeln zu kommunizieren.

Eine weitere Kernerkenntnis der Untersuchung ist, dass Homeoffice - im Gegensatz zu Rückzugsorten innerhalb der Unternehmen - als passend für konzentriertes Arbeiten eingeschätzt wird. Im Vergleich zu Rückzugsorten innerhalb des Unternehmens besteht im Homeoffice keine tatsächliche bzw. keine auch nur gefühlte Konkurrenz um diese Rückzugsorte, was der Konzentration förderlich sein kann. Eine Behauptung, die mit den Ergebnissen dieser Studie vereinbar ist, wäre es, dass ActivityBased Flexible Offices mit Homeoffice-Konzepten kombiniert werden müssen. In einigen Unternehmen ist dies wohl die Wirklichkeit. Unseres Erachtens sollte das Hauptergebnis dieser Studie als Hinweis aufgegriffen werden: Ist ein Activity-Based Flexible Office nicht richtig umgesetzt, weil z.B. Rückzugsorte fehlen oder es an der individuellen oder kollektiven Kompetenz zur adäquaten Raumnutzung fehlt, so kann eine Folge sein, dass Mitarbeitende für konzentriertes Arbeiten das Homeoffice aufsuchen. Ob und in welchem Ausmaß das erwünscht ist, sollte vor der Einführung einer neuen Arbeitswelt strategisch erwogen werden.

\section{Methodische Einschränkungen}

Wie jede Studie weist auch die vorliegende Studie methodische Einschränkungen auf. Erstens fußen einige der Maße wie die Konzentrationserfordernisse und die wahrgenommene Passung auf jeweils einem Item. Dieses wird den psychometrischen Anforderungen an die Erhebung von Konstrukten nicht vollkommen gerecht; allerdings sollte die Itemanzahl bei täglichen Befragungen auf ein Mindestmaß reduziert werden. Ohly, Sonnentag, Niessen und Zapf (2010) schlagen vor, jeweils ein Item pro Konstrukt auszuwählen, welches dieses am besten repräsentiert.

Zweitens handelt es sich bei den erhobenen Maßen um Selbstauskünfte, so dass ein common method bias vorliegen könnte. Eine weitere Erhebungsform wäre eine Fremdeinschätzung, was hinsichtlich der Arbeitsaufgabe und des Arbeitsortes eine sinnvolle Ergänzung dargestellt hätte. Da es sich bei der wahrgenommenen Passung und noch mehr beim psychischen Wohlbefinden um subjekti- ve Einschätzungen handelt, war es bei diesen Maßen alternativlos, die Beschäftigten als Betroffene zu fragen.

Drittens könnte die wiederholte Befragung die Teilnehmenden sensibilisiert haben: Da die Teilnehmenden wiederholt nach der Passung von Arbeitsaufgabe und Arbeitsort befragt wurden, könnten diese an den folgenden Tagen verstärkt darauf geachtet haben, einen passenden Arbeitsort zu wählen, was Einfluss auf die Ergebnisse gehabt haben könnte. Allerdings scheinen die verzerrenden Effekte des sog. panel conditioning - die Veränderung des zu Messenden durch seine wiederholte Messung - gering auszufallen bzw. sogar die Validität zu steigern (Struminskaya \& Bosnjak, 2021).

Viertens könnte bei dieser längsschnittlichen Untersuchung theoretisch eine selektive Mortalität der Teilnehmenden aufgetreten sein. Dieses Problem ist bei den vorliegenden Daten wenig wahrscheinlich bzw. von geringem Ausmaß, da teilweise Erhebungen ausgelassen wurden, die Personen sich aber zu späteren Untersuchungszeitpunkten wieder beteiligten.

Schließlich wurde der Fragebogen immer am Ende eines Arbeitstages ausgefüllt und die Beantwortung der Fragen bezogen sich auf die Tätigkeiten der letzten zwei Stunden. Die Arbeitsaktivitäten, welchen die untersuchten Beschäftigten eher gegen Ende des Tages nachgingen, könnten sich systematisch von Aufgaben z. B. am Beginn des Arbeitstages unterscheiden. Obwohl wir Unterschiede in den Konzentrationsanforderungen festgestellt haben, könnten zukünftige Studien den Aspekt Tageszeit näher untersuchen bzw. mehrfache Erhebungen am Tag vorsehen.

\section{Implikationen und Ausblick}

Die vorliegende Untersuchung verdeutlicht die Bedeutungshaftigkeit von offenen Bürokonzepten für die psychische Gesundheit, insbesondere das psychische Wohlbefinden. Damit trägt die Studie zur bisherigen Forschung bei, da bisher nur wenige quantitative Studien zum Thema Activity-Based Flexible Offices und psychisches Befinden vorliegen (Engelen et al., 2018; Richardson et al., 2017). Insbesondere fehlt es an Längsschnittstudien, einer mehrdimensionalen Erfassung von Büroräumen („Kein Büroraum ist wie der andere.") sowie einer interdisziplinären Betrachtung des Themas (Lütke Lanfer \& Pauls, 2017). Die vorliegende Tagebuchstudie legt erste empirische Erkenntnisse zur Bedeutung einzelner Arbeitsaufgaben für die erlebte Passung zwischen Konzentrationserfordernissen und Arbeitsort einerseits und für das psychische Wohlbefinden andererseits dar. Es zeigte sich insbesondere, dass die Erfordernisse der einzelnen Arbeitsauf- 
gaben bei der flexiblen Arbeitsortwahl berücksichtigt werden sollten.

Bei der Umsetzung neuer Arbeitswelten gibt es unzählige Varianten und Einflussgrößen, die allgemeingültige Aussagen fast unmöglich machen. Das erklärt womöglich auch, warum die Befunde zum Zusammenhang zwischen Arbeitsplatzkonzepten und psychischem Wohlbefinden so uneinheitlich ausfallen. Für Praktiker ist es unumgänglich, neue Arbeitswelten immer wieder neu zu erproben, wie es Bernstein und Waber (2019) erkannt haben: Ein Patentrezept für das richtige Activity-Based Flexible Office gibt es nicht. Es ist denkbar, dass Beschäftigte eine gewisse Zeit benötigen, um Erfahrungen in der Auswahl passender Arbeitsorte $\mathrm{zu}$ sammeln und um passende Routinen zu entwickeln. Aus einer übergeordneten Perspektive heraus, geht das Konzept des Activity-Based Flexible Office darüber hinaus und ist als ein kontinuierlicher Prozess zu verstehen, d.h. Organisationen müssen immer wieder ausloten, welche Arbeitsweisen mit welchen Arbeitsumwelten funktionieren. Das gilt auch für das Thema Konzentration in Activity-Based Flexible Offices.

\section{Fazit}

Das Activity-Based Flexible Office sollte als ganzheitliches Konzept verstanden werden, in dem Rückzugsorte innerhalb des Unternehmens sowie das Homeoffice als alternative Arbeitsorte mitgedacht werden. Activity-Based Flexible Offices sollten die Freiheit bieten, unterschiedliche Aufgaben an unterschiedlichen Orten ausführen zu können. Aber diese Freiheit allein reicht nicht aus - für Praktiker ist es unumgänglich, neue Arbeitswelten immer wieder neu zu erproben und anzupassen, sonst kann die Freiheit zur Überforderung werden.

\section{Literatur}

Appel-Meulenbroek, R., Groenen, P. \& Janssen, I. (2011). An enduser's perspective on activity-based office concepts. Journal of Corporate Real Estate, 13, 122 -135. https://doi.org/10.1108/ 14630011111136830

Becker, C., Lütke Lanfer, S. S. \& Kratzer, N. (2019). Neue Arbeitswelten: Wahrnehmung und Wirkung von Open-Space-Büros. Arbeit, 28, 263 - 284. https://doi.org/10.1515/arbeit-2019-0017

Bernstein, E. \& Waber, B. (2019). The truth about open offices. Harvard Business Review, 97, 82 - 91

Bodin Danielsson, C. \& Bodin, L. (2008). Office type in relation to health, well-being, and job satisfaction among employees. Environment and Behavior, 40, 636-668. https://doi.org/10.1177/ 0013916507307459

Bodin Danielsson, C., Singh Chungkham, H., Wulff, C. \& Westerlund, H. (2014). Office design's impact on sick leave rates. Er- gonomics, 57, 139-147. https://doi.org/10.1080/00140139 2013.871064

Brähler, E., Mühlan, H., Albani, C. \& Schmidt, S. (2007). Teststatistische Prüfung und Normierung der deutschen Versionen des EUROHIS-QOL-Lebensqualität-Index und des WHO-5 Wohlbefindens-Index. Diagnostica, 53, 83-96. https://doi.org/10. 1026/0012-1924.53.2.83

Bundesanstalt für Arbeitsschutz und Arbeitsmedizin (2016). Arbeitswelt im Wandel: Zahlen - Daten - Fakten. Dortmund: Bundesanstalt für Arbeitsschutz und Arbeitsmedizin.

Caplan, R. D. (1987). Person-environment fit theory and organizations: Commensurate dimensions, time perspectives, and mechanisms. Journal of Vocational Behavior, 31, 248-267. https://doi.org/10.1016/0001-8791(87)90042-x

Cascio, W. F. \& Montealegre, R. (2016). How technology is changing work and organizations. Annual Review of Organizational Psychology and Organizational Behavior, 3, 349-375. https://doi. org/10.1146/annurev-orgpsych-041015-062352

Davis, M. C., Leach, D. J. \& Clegg, C. W. (2011). The physical environment of the office: Contemporary and emerging issues. In G. P. Hodgkinson \& J. K. Ford (Eds.), International Review of Industrial and Organizational Psychology (pp. 193-237). Hoboken, NJ: Wiley-Blackwell. https://doi.org/10.1002/97811199 92592.ch6

De Been, I. \& Beijer, M. (2014). The influence of office type on satisfaction and perceived productivity support. Journal of Facilities Management, 12, 142 -157. https://doi.org/10.1108/ JFM-02-2013-0011

De Croon, E., Sluiter, J., Kuijer, P. P. \& Frings-Dresen, M. (2005). The effect of office concepts on worker health and performance: A systematic review of the literature. Ergonomics, 48, 119 - 134. https://doi.org/10.1080/00140130512331319409

Edwards, J. R., Cable, D. M., Williamson, I. O., Lambert, L. S. \& Shipp, A. J. (2006). The phenomenology of fit: Linking the person and environment to the subjective experience of person-environment fit. Journal of Applied Psychology, 91, 802-827. https://doi.org/10.1037/0021-9010.91.4.802

Edwards, J. R., Caplan, R. D. \& Harrison, R. V. (1998). Person-environment fit theory: Conceptual foundations, empirical evidence, and directions for future research. In C. L. Cooper (Ed.), Theories of organizational stress (pp. 28-67). Oxford: Oxford University Press.

Elsbach, K. D. \& Pratt, M. G. (2007). The physical environment in organizations. The Academy of Management Annals, 1, $181-$ 224. https://doi.org/10.1080/078559809

Engelen, L., Chau, J., Young, S., Mackey, M., Jeyapalan, D. \& Bauman, A. (2018). Is activity-based working impacting health, work performance and perceptions? A systematic review. Building Research \& Information, 47, 468-479, https://doi.org/10.1080/ 09613218.2018 .1440958

Gerdenitsch, C., Korunka, C. \& Hertel, G. (2018). Need-supply fit in an activity-based flexible office: A longitudinal study during relocation. Environment and Behavior, 50. 273 -297. https://doi. org/10.1177/0013916517697766

Heerwagen, J. H., Kampschroer, K., Powell, K. M. \& Loftness, V. (2004). Collaborative knowledge work environments. Building Research \& Information, 32, 510 - 528. https://doi.org/10.1080/ 09613210412331313025

Herbig, B., Schneider, A. \& Nowak, D. (2016). Gesundheit, Kommunikation und Leistung in Großraumbüros - Zusammenhänge mit Personenzahl, spezifischen Umgebungsbedingungen und allgemeinen Tätigkeitsmerkmalen. Wirtschaftspsychologie, 18, $71-81$.

Imai, K., Keele, L. \& Tingley, D. (2010). A general approach to causal mediation analysis. Psychological Methods, 15, 309-334. https://doi.org/10.1037/a0020761 
Kratzer, N. (Hrsg.). (2017). Open Space. Oder was? Wandel der Büroarbeit. München: ISF München.

Kratzer, N. (Hrsg.). (2020). Open Space. Besser machen. Eine Praxisbroschüre des Projekts PRÄGEWELT - „Präventionsorientierte Gestaltung neuer Open-Space-Arbeitswelten“. München: ISF München.

Kristof, A. L. (1996). Person-organization fit: An integrative review of its conceptualizations, measurement, and implications. Personnel Psychology, 49, 1 -49. https://doi.org/10.1111/j.17446570.1996.tb01790.x

Lütke Lanfer, S. S., Becker, C. \& Göritz, A. S. (2021). Well-being in Open Space Offices: The role of office features and psychosocial working conditions. Work, 68, 317-332.

Lütke Lanfer, S. S. \& Pauls, N. (2017). Eine systematische Literaturrecherche zu modernen Bürostrukturen und deren Auswirkung auf psychisches Befinden. In Gesellschaft für Arbeitswissenschaft (Hrsg.), Soziotechnische Gestaltung des digitalen Wandels - kreativ, innovativ, sinnhaft. Dortmund: GfA-Press.

Ohly, S., Sonnentag, S., Niessen, C. \& Zapf, D. (2010). Diary studies in organizational research. Journal of Personnel Psychology, 9 , 79-93. https://doi.org/10.1027/1866-5888/a000009

Richardson, A., Potter, J., Paterson, M., Harding, T., Tyler-Merrick, G., Kirk, R., Reid, K. \& McChesney, J. (2017). Office design and health: A systematic review. New Zealand Medical Journal, 130, $39-49$

Richter, G. \& Cernavin, O. (2016). Büro als Treiber gesundheitsförderlicher und produktiver Arbeitsbedingungen. In M. Klaffke (Hrsg.), Arbeitsplatz der Zukunft (S. 81-101). Wiesbaden: Springer.

Rothe, I., Adolph, L., Beermann, B., Schütte, M., Windel, A., Grewer, A., Lenhardt, U., Michel, J., Thomson, B. \& Formazin, M. (2017). Psychische Gesundheit in der Arbeitswelt. Wissenschaftliche Standortbestimmung. Dortmund: Bundesanstalt für Arbeitsschutz und Arbeitsmedizin.

Seddigh, A., Berntson, E., Bodin Danielson, C. \& Westerlund, H. (2014). Concentration requirements modify the effect of office type on indicators of health and performance. Journal of Environmental Psychology, 38, 167 -174. https://doi.org/10.1016/j. jenvp.2014.01.009

Semmer, N., Zapf, D. \& Dunckel, H. (1999). Instrument zur Stressbezogenen Tätigkeitsanalyse (ISTA). In H. Dunckel (Hrsg.), Handbuch psychologischer Arbeitsanalyseverfahren. Zürich: vdf Hochschulverlag.

Singer, J. D. \& Willett, J. B. (2003). Applied longitudinal data analysis: Modeling change and event occurrence. New York: Oxford University Press.

Stadler, S. (2011). Open Space Büros: Eine Studie über die Machbarkeit und Umsetzung von offenen Bürostrukturen. Düsseldorf: Hans-Böckler-Stiftung. https://www.boeckler.de/pdf_fof/9733 7.pdf

Struminskaya, B. \& Bosnjak, M. (2021). Panel conditioning: Types, causes and empirical evidence of what we know so far (pp. 272-301). Hoboken, NY: Wiley. https://doi.org/10.1002/ 9781119376965.ch12

Thörel, E., Pauls, N. \& Göritz, A. S. (2020). Antezedenzien und Wirkmechanismen arbeitsbezogener erweiterter Erreichbarkeit - Wenn die Arbeitszeit nicht ausreicht. Gruppe. Interaktion. Organisation. Zeitschrift für Angewandte Organisationspsychologie. https://doi.org/10.1007/s11612-020-00496-z
Utsch, C. (2017). In der Schwebe. Produktives Arbeiten im digitalisierten Zeitalter. ZWF Zeitschrift für wirtschaftlichen Fabrikbetrieb, 112, 410-412. https://doi.org/10.3139/104.111742

Van der Voordt, T. J. M. (2004). Productivity and employee satisfaction in flexible workplaces. Journal of Corporate Real Estate, 6, 133 -148. https://doi.org/10.1108/14630010410812306

Windlinger, L., Konkol, J., Schanné, F., Sesboüé, S. \& Neck, R. (2014). Gesundheitsförderliche Büroräume. Wissenschaftliche Grundlagen zum Zusammenhang zwischen psychischer Gesundheit und Büroraumgestaltung sowie dem begleitenden Veränderungsprozess. Bern und Lausanne: Gesundheitsförderung Schweiz. https://digitalcollection.zhaw.ch/handle/11475/6343

Wohlers, C., Hartner-Tiefenthaler, M. \& Hertel, G. (2017). The relation between activity-based work environments and office workers' job attitudes and vitality. Environment and Behavior, 51, 167 -198. https://doi.org/10.1177/0013916517738078

Wohlers, C. \& Hertel, G. (2017). Choosing where to work at work Towards a theoretical model of benefits and risks of activitybased flexible offices. Ergonomics, 60, 467-486. https://doi. org/10.1080/00140139.2016.1188220

\section{Historie}

Eingegangen: 08.03.2020

Revision eingegangen: 29.01.2021

Onlineveröffentlichung: 08.06. 2021

\section{Danksagung}

Universität Freiburg: Leandra Lemmer, Nina Pauls, Milena Gehlert; PGW Team: v. a. Nick Kratzer, Alexandra Frot

\section{Autorenschaften}

Geteilte Erstautorenschaft der beiden erstgenannten Autoren. Die Verantwortung für den Inhalt dieser Veröffentlichung liegt bei den Autoren.

\section{Förderung}

Die Untersuchung wurde im Rahmen des Projektes „PRÄGEWELT - präventionsorientierte Gestaltung neuer Open-Space-Arbeitswelten“ durchgeführt. Dieses Forschungsprojekt wurde mit Mitteln des Bundesministeriums für Bildung und Forschung (BMBF) im Programm „Innovationen für die Produktion, Dienstleistung und Arbeit von morgen" (FKZ 02 L14 A101) gefördert und vom Projektträger Karlsruhe (PTKA) betreut (Laufzeit: 01.01.2016 bis 31.07. 2019). Mehr dazu unter www. praegewelt.de.

Open Access-Veröffentlichung ermöglicht durch die FriedrichAlexander-Universität Erlangen-Nürnberg.

\section{ORCID}

Roman Soucek

(iD) https://orcid.org/0000-0002-9877-1414

\section{PD Dr. Roman Soucek}

Lehrstuhl für Psychologie

insbes. Wirtschafts- und Sozialpsychologie

Friedrich-Alexander-Universität Erlangen-Nürnberg

Lange Gasse 20

90403 Nürnberg

roman.soucek@fau.de 